\title{
Implementation and Usage of an Online Environment in a Chemical Engi- neering Curriculum
}

\section{Kyle Joe Branch, University of Utah}

Kyle Branch is a third-year graduate student at the University of Utah Department of Chemical Engineering. He has helped develop and teach two freshman courses, using the materials and methods described in this paper. His main research interest is in engineering education, focusing on the creation and analysis of interactive simulations for undergraduate chemical engineering courses.

\section{Prof. Anthony Butterfield, University of Utah}

Anthony Butterfield is an Assistant Professor (Lecturing) in the Chemical Engineering Department of the University of Utah. He received his B. S. and Ph. D. from the University of Utah and a M. S. from the University of California, San Diego. His teaching responsibilities include the senior unit operations laboratory and freshman design laboratory. His research interests focus on undergraduate education, targeted drug delivery, photobioreactor design, and instrumentation. 


\title{
Implementation and Usage of an Online Environment in a Chemical Engineering Curriculum
}

\begin{abstract}
We have developed an online system to serve as a hub for student activities in our chemical engineering courses. In addition to generating and grading textbook-style homework problems, it provides interactive simulations of laboratory experiments, and it administers surveys, pre- and post-tests, and quizzes for training on laboratory equipment. The system randomizes the numbers and units in homework problems and allows multiple attempts, which discourages cheating, removes the burden of manual grading for instructors, and provides students with instantaneous feedback. Students complete assignments using simulated laboratory experiments, which model corresponding hands-on projects that they will later work on in class. This is done to familiarize students with the project and core chemical engineering theory ahead of time, saving lab resources and time and enabling students to work at their own pace.

In order for students to become certified to use particular laboratory equipment, they must pass an online quiz on the safety and operating procedures of the equipment before training with an instructor. This saves instructor time and allows students to go back and review the material at any time. Students are able to print out their name badge from the site, which includes a list of all of the equipment they have been certified to use. This feature helps instructors learn the students' names and allows instructors to easily check if students are certified to use specific equipment.
\end{abstract}

In analyzing data collected by the online system, we discovered differences in behavior between higher- and lower-performing students. Based on this information, we have offered our students advice on study habits that are associated with better performance. We have also used this data to identify and address common student misunderstandings.

We have also added game-like aspects to the system: as students progress through assignments and become certified to use laboratory equipment, they "gain experience" and "level up" on the website. Such techniques have been shown to increase student engagement. Since the addition of these features, we have observed students completing unassigned problems and trying to become trained on as much equipment as possible.

In order to ensure that the system is as easy to use as possible, we have made it open-source and freely accessible to instructors and students alike. Additionally, it is designed to work on all major browsers, enabling its use on all platforms and devices, without requiring the download or installation of any files.

\section{Introduction}

A meta-analysis of more than 1000 articles has shown that students in higher-education, on average, perform better using online learning techniques than those receiving face-to-face interactions ${ }^{1}$. Additionally, there has been no significant difference found in the quality of student retention of scientific concepts between those using online and hands-on learning techniques ${ }^{2}$. In order to cater to as many learning styles as possible, we have developed a 
freshman design laboratory course, in the Department of Chemical Engineering at the University of Utah, in which we have coupled traditional, virtual, and hands-on learning techniques, detailed in $^{3,4}$.

For this course, we have developed a website to act as an online hub for the students in our department. This system has been designed, in particular, to cater with the increasingly large class sizes we have seen in this course, which have been a nation-wide trend engineering courses in recent years ${ }^{5}$. This online system is located at vSTEM.org, which stands for virtual STEM (Science, Technology, Engineering, and Mathematics). We have created this website to provide our students with various course materials, from interactive simulations to safety training quizzes for equipment in the lab. We have developed the website to be open-source and freely accessible to students and faculty at other universities. It has been designed to be easily adopted into other departments and universities, and faculty at other universities have already found our simulations using search engines, and used them in classroom demonstration.

\section{Methods}

Our website, vSTEM, has been created using PHP, HTML, and Javascript, to eliminate the need for students to download or run any external files, which they might be hesitant or unable to do. Additionally, vSTEM is accessible and supported on all major browsers, students are able to access it on any internet-enabled device, from smart phone to laptop.

Occasionally, students are asked to complete surveys or to provide demographic data to be used in the analysis, which is all optional. To ensure all student information is secured, it is stored in a password-protected MySQL database. Student passwords are encrypted, and student-submitted information is only displayed and analyzed in ensemble averages.

\section{Homework Assignments}

Our primary use of vSTEM is to assign homework problems, which are automatically graded, giving the students instantaneous feedback. This helps remove the burden of grading on instructors and teaching assistants, freeing them up to help students one-on-one. The website has been designed to give the instructors as much control over the problem statements as possible, in order to achieve whatever type of functionality is desired, while still being easy to use.

With vSTEM, we are able to assign textbook-style homework problems with randomized values for the variables. This is done so that every student has a slightly different problem to solve, discouraging cheating. The instructor can determine the points and number of attempts each student is allowed for each problem. Additionally, the instructor can set up questions so that each student has to solve for a randomly selected unknown variable, as opposed to every student solving for the same variable. This gives students a more unique problem to solve, and, if they solve the problem incorrectly, their next attempt may ask them to solve for something slightly different. The instructor can also set up problems to use randomized units - they can choose be metric, English, or a mixture of both. For assignments aimed at teaching unit conversions, there is also an option for "messy" units, which includes many unit types that are uncommonly used. 
Finally, the instructors can define a late penalty, which will automatically be applied to any problems which are submitted after the deadline. Currently, the late penalty must be in the form $A-B \cdot \exp (-C t)$, where $A, B$, and $C$ are parameters set by the instructor, and $t$ is how late the student submits the assignment. The instructors can easily give a single student a permanent extension on all assignments, which may be used to accommodate students with recognized learning disabilities. The instructor can set a closure date, after which the students can no longer submit answers for a particular assignment.

Besides assigning textbook-style problems, the system can assign multiple-choice questions. The instructor can change whether or not the students can select multiple answers, and whether the order of the selections is to be randomized. They can also ask Likert-style survey questions; for these, the instructor can alter the header of each column, which makes them useful for a wide variety of applications, including pre- or post-course surveys. If the instructor allows, the students will be able to see the correct answer if they are wrong, or go back and view all of their previous submissions for a problem.

If an instructor would like to mimic a standard assignment, they could require all questions to be submitted at the same time and prevent the students from receiving instantaneous feedback. We have used this functionality to replace hand-written pre- and post-tests used for assessing student learning, while keeping them as similar as possible to their hand-written counterpart in order for them to be used in course benchmarking. Also, for assignments like these, instructors have the option of simply assigning them as "credit" or "no credit", based on whether or not the student submits it.

In addition to these static problems, we also created a variety of interactive simulations, each designed to mimic a specific laboratory environment. For example, there is a simulation of a bioreactor, with adjustable parameters, such as the initial concentration of cells, substrate, toxins, dissolved gas, the cell's maximum specific growth rate, etc. Students may adjust these parameters and then start the bioreactor and watch the cells traverse through the lag, exponential growth, stationary, and death phases. In our interactive simulations, each of the parameters can be hidden from the student, and the student's task can be to determine it within a sufficient degree of accuracy.

In our freshman design lab course, we lecture on the basics of the current week's topic and then assign our students to use these simulations, to ensure they understand the material. Afterwards, we assign them to build a physical apparatus in the lab, as a team, mimicking the one in the simulation. They are then tasked with determining the same unknown parameters with their data as they determined in the simulation. For example, we will have the students use a spectrophotometer simulation to determine chemical parameters such as a molecule's molar extinction coefficient and a reaction rate constant, after which they build a spectrophotometer in the lab and experimentally determine the same parameters.

These simulations are designed to help the students gain an understanding of core concepts before working in the lab, in order to save lab resources and time. Having the students work on the simulations alone, before working on the project as a team also enables the students to work 
at their own pace, and improves the design of their physical apparatus, as they experience some common problems and brainstorm ideas of how to build the apparatus beforehand.

The interactive simulations record all student interactions, including mouse movements and locations, clicks, keystrokes, field alterations, etc. which have been analyzed and discussed in ${ }^{6}$.

\section{Lab Safety and Equipment Training}

Our laboratory is equipped with additive and subtractive prototyping equipment, from simple dremels and a drill press to laser cutters and 3D printers. We allow our students to use this equipment for class projects or their undergraduate research, as long as they have been properly trained and the cost of the materials is reasonable.

In the past, we have required each student to be individually trained, verbally quizzed, and then observed operating any piece of equipment they desired to use. Due to our large class sizes, this became an increasingly difficult task. In order to help streamline the training process, we have added equipment safety and operating procedure training quizzes on vSTEM, which we require the students to complete before meeting with us to train on a piece of equipment. In order for a student to complete the online training quiz for a particular piece of equipment, they must get every question correct. If they incorrectly answer any of the problems, they will have to retake the quiz again; they can do this as many times as necessary.

These training quizzes not only reduce the amount of time required to train and quiz each student, but also gives the students a break between being quizzed on the equipment online and in person, which has been shown to help students retain the information better ${ }^{7}$ and hence improve the safety of the lab. Additionally, students are always able to go back and view the quiz with their answers at any time if they forgot any of the material.

In order to improve safety in the lab, we also ask students to wear a name badge which shows all of the equipment they are certified to use. These not only help the instructors learn the students' names, but also helps the instructors and lab manager quickly see if a student has been trained on a piece of equipment from a glance. All student training is recorded online, and students are able to print out a new name badge on VSTEM if they become certified to use another piece of equipment, or need a replacement.

\section{Gaming Aspects}

The website also has many features that are commonly found in video games. Game-like aspects within a course have been shown to improve student learning and attitudes towards the course in a meta-analysis of 62 articles $^{8}$. On vSTEM, students are able to gain "imaginary experience points" by completing their homework assignments and by becoming certified to use new pieces of lab equipment. Students are able to complete unassigned homework problems to gain more experience points, however, these extra problems and experience points do not benefit the student or their grade in any way. As students gain these experience points, they can "level up". Each level has a unique and creative title that has been designed to entertain the students. For example, when they start out they are just a "Duckling", when they reach level 6 they become a "Terran SCV", and at level 15, their title is "Maxwell's Demon". 


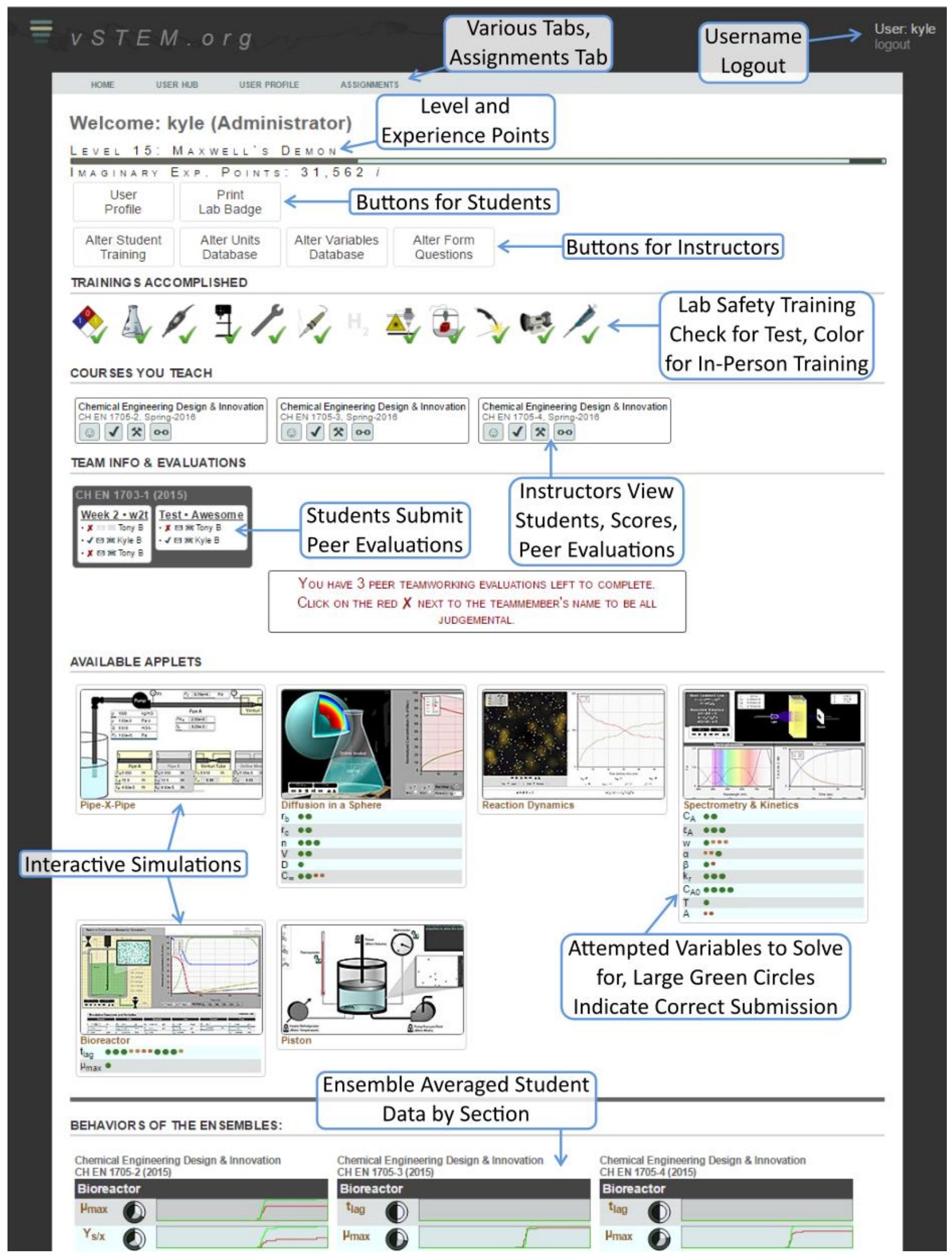

Figure 1: A screenshot of the User Hub on vSTEM.org, as seen by an administrator. The website looks very similar as a student or instructor, with a few icons changed, as distinguished in the figure. 


\section{Team-working Evaluations}

Finally, we use vSTEM to create student teams. For each course project we assign teams of three for students to work in. Projects typically last one to two weeks. Using vSTEM, students are able to see who their team members are and share their email address or phone number with their team if they desire. This can help students contact each other if they need to work on their project outside of class time. Additionally, students are required to submit peer evaluations of their team members after each project. Using a form on vSTEM, they can submit a quick evaluation survey for each of their team members. This information is stored in a database in a form that can be easily analyzed by the instructor.

\section{vSTEM Website}

A screenshot of what the website looks like is shown in Figure 1, with a brief description of what each section of the website is for. If the student is currently or previously enrolled in any course that has assignments, the student will be able to see the Assignments Tab and go to the Assignments page. A labeled screenshot of the Assignments page is shown in Figure 2. The students are always able to go back and see any of their previous assignments, unless the assignment is closed and was set to not give the students feedback. If the students have any assignments which are late, not yet closed, and still have problems left to submit, then the last

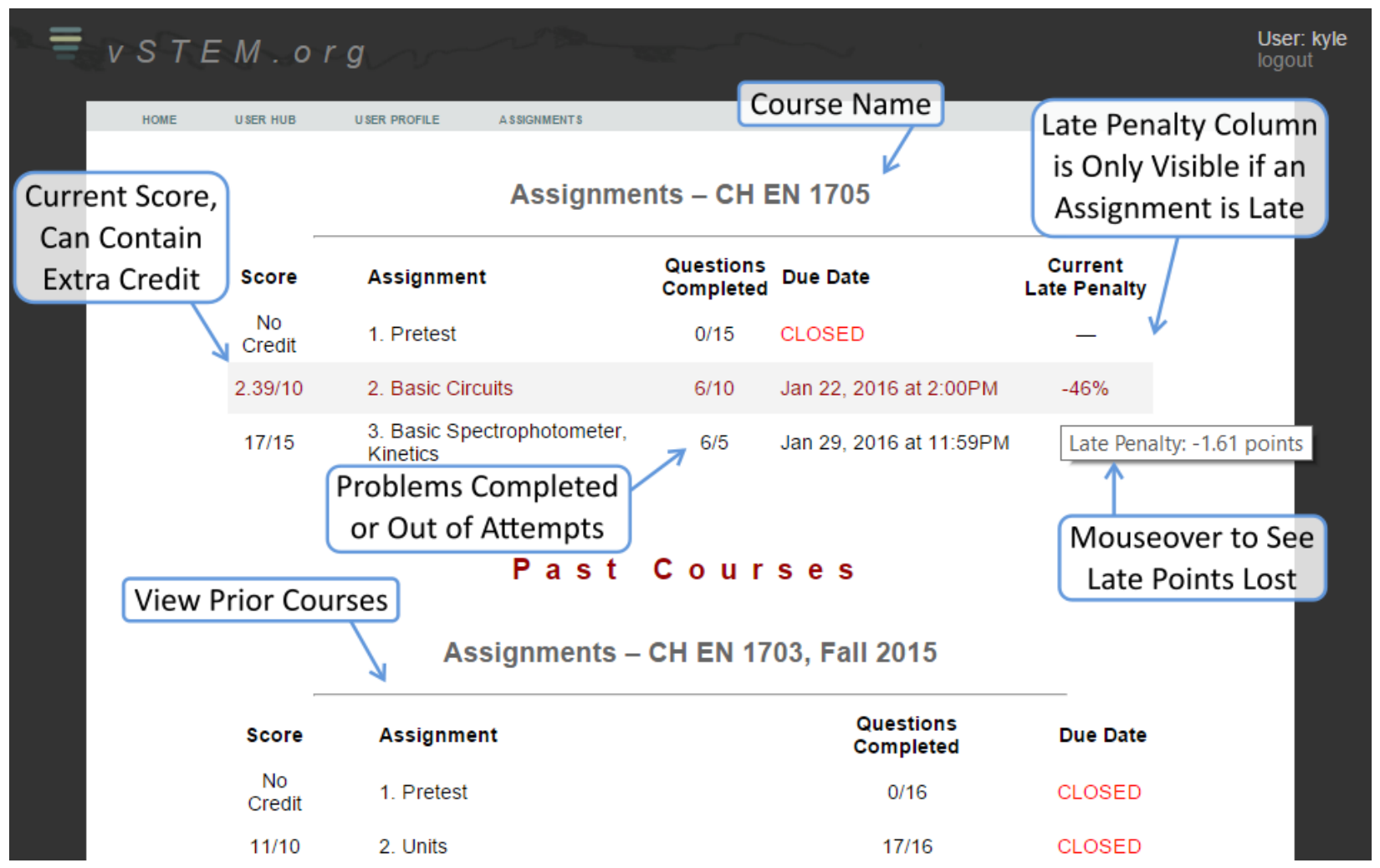

Figure 2: A screenshot of the Assignments Tab on vSTEM.org. Students must be enrolled or previously enrolled in at least one course with assignments in order to view the Assignments Tab. Students can click on any assignment to view the assignment, submit answers, or view their previous submissions. 
column will be shown, which displays to the students the current late penalty for submitting more problems in that assignment.

\section{Results}

In all, we feel that the website has been a great addition to our course. Having the assignment automatically graded significantly reduces required time grading for the instructors and teaching assistants. This helps free up our time to help students more in the lab, and individually mentor them when they fall behind.

We have also been able to use the interactive simulations' tracking capabilities to determine some common differences in student behavior between our higher- and lower-performing students ${ }^{6}$. In this analysis, we have found that students who complete their homework earlier in the week, and even earlier in the day perform better than those who wait until right before an assignment is due, and those who stay up late at night working on their homework. In our classes, we have begun showing the students some of the data we have collected and describing the behaviors that we have found to correlate with student success. With this we hope to help the students develop study habits which will help them succeed.

Using this tracking data, we are able to play-back individual student mouse locations as they completed their homework problems, as shown in Figure 3. We have used this functionality to determine individual student misconceptions, as well as to determine and implement improved user-interface functionality. In this analysis, we have also been able to determine some common student misunderstandings using the differences between ensemble-averaged mouse location data for correct and incorrect submissions. These common problems can then be addressed by emphasizing them in the lecture.

For the interactive assignments, we have typically given the students an unlimited number of attempts. We have determined several characteristics of student guessing, and have told our students that they will not get credit if they are determined to be guessing. Even after telling them, we still found a significant amount of student guessing. We found that those who stay up late working on an assignment the night before it is due are far more likely to simply repeatedly guess answers, as opposed to attempt to truly learn the material. In order to remedy this, we are now moving to a new format, in which the students have an unlimited number of practice attempts, but only a few attempts for credit.

In addition to the benefits gained from using vSTEM's homework system, we have found the online safety training quizzes to be helpful in the lab. Having students take these quizzes before being trained on lab equipment has reduced the amount of time it takes for an instructor to train students on lab equipment. This has helped make the first few weeks of lab more manageable, as the instructors can spend more time helping students with their projects and students can spend less time waiting in line to be trained on commonly used equipment, such as the drill press. 


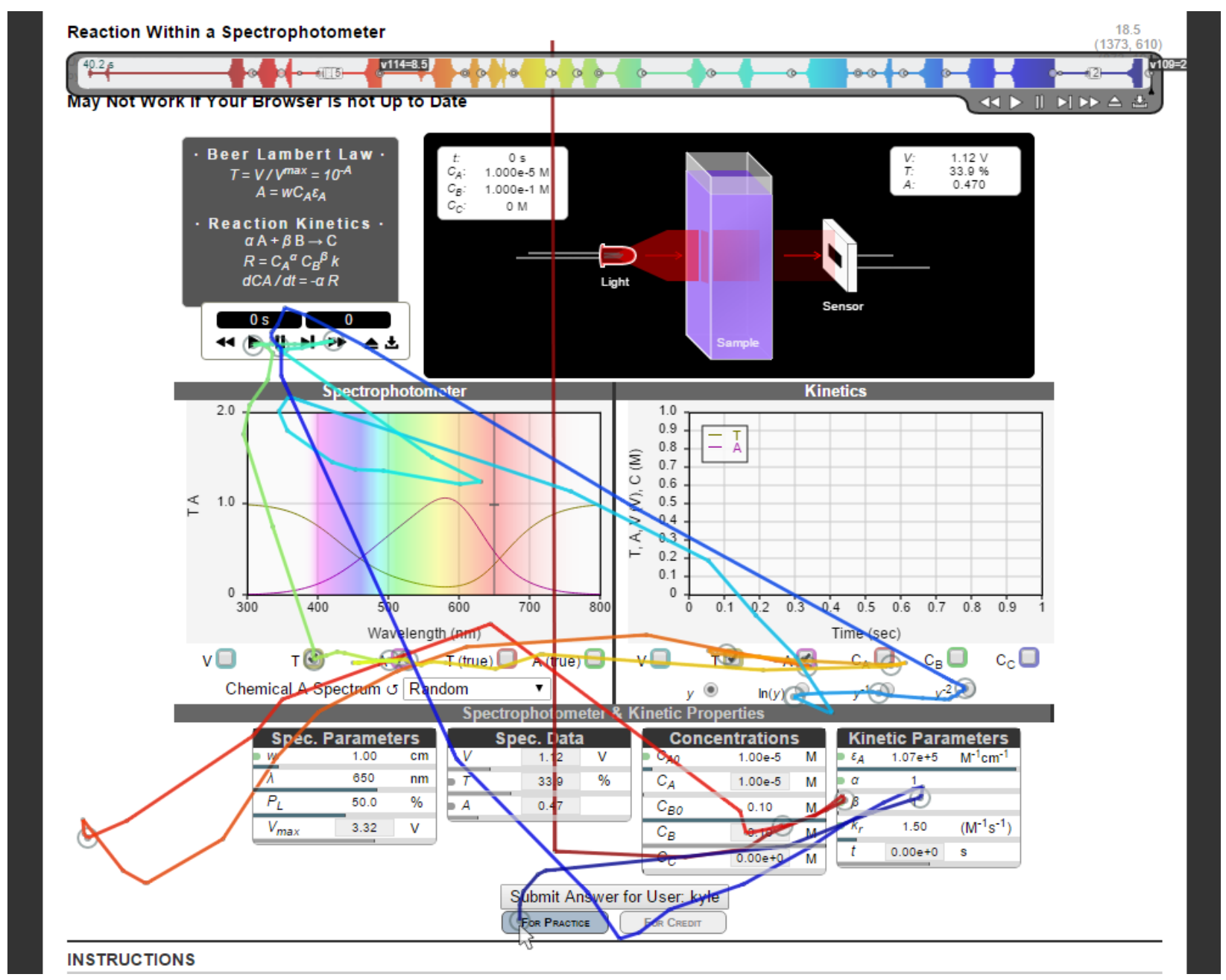

Figure 3: Instructors are able to view a video of an individual student's attempt on a homework problem. The bar at the top will allow the instructor to play and rewind the video. The thickness of the play bar indicates mouse movement speed at that time interval. During the video, and on the play bar, there are indications of student mouse-clicks and key-strokes.

Finally, several students have shown appreciation for the game-like aspects added to vSTEM. Even though gaining these imaginary experience points does not give the students any benefit in the course, a few students noticed the leveling system and learned how to solve unassigned homework problems just to gain this experience. This semester we have more actively informed the students of this system, and have noticed an increase in the number of students attempting to gain as much experience as they can. Several students have told us that they were trying to level up just to see the title of the next level, or to reach a higher level than their friends and peers.

\section{Conclusions}

We have developed an online hub to contain a variety of materials for use in our courses. This system is used as a means to generate, grade, and provide instantaneous feedback for students' homework assignments. In addition to static textbook-style homework problems, we have created a variety of interactive simulations that are designed to mimic experimentation in a 
laboratory setting. This homework system was designed to save the instructor's time by eliminating the need for grading, allowing them to spend more time helping students with individualized misunderstandings.

In addition to homework assignments, we use this website to provide safety and operating procedure training quizzes, and track student training. Students are required to wear a name badge in the lab, which they can print out from their account on the website. This name badge has a symbol for every piece of equipment that they have been certified to use, allowing the lab manager and instructors to easily check, from a glance, if a student is allowed to use the equipment.

Finally, we have started using this system to assign student laboratory teams. Students can easily see who is in their team for the current project, and share their contact information if they desire. On this website, students are also required to submit peer evaluations for their team members, which the instructor can easily view.

\section{References}

[1] Means, Barbara, et al. "Evaluation of evidence-based practices in online learning: A meta-analysis and review of online learning studies." US Dept. of Educ., 2009.

[2] M. D. Renken and N. Nunez, "Computer simulations and clear observations do not guarantee conceptual understanding," Learn. Instr., vol. 23, pp. 10-23, 2013.

[3] A. Butterfield, K. Branch, E. Trujillo, "First-Year Hands-On Design Course: Implementation \& Reception," Chem. Eng. Ed., vol. 49, no. 1, pp. 19-26 2014.

[4] A. Butterfield and K. Branch, "Results \& Lessons Learned from a Chemical Engineering Freshman Design Laboratory," 2015 ASEE Annu. Conf. Expo., 2015.

[5] B. Yoder, "Engineering by the Numbers," ASEE, 2014.

[6] K. Branch, and A. Butterfield, "Analysis of Student Interactions with Browser-Based Interactive Simulations," Comp. in Educ. J., in press, 2015.

[7] Lieb, Stephen, and John Goodlad. "Principles of adult learning." (2005).

[8] Bodnar, Cheryl A., et al. "Engineers at Play: Games as Teaching Tools for Undergraduate Engineering Students." J. of Eng. Educ., 2016. 